\title{
2013 Colorado alphaherpesvirus latency symposium
}

\author{
Randall J. Cohrs • Don Gilden
}

Received: 10 September 2013 / Accepted: 19 September 2013 /Published online: 3 October 2013

(C) Journal of NeuroVirology, Inc. 2013

The third symposium of the Colorado Alphaherpesvirus Latency Society (CALS) convened May 15-17, 2013, at the Christiania Lodge in Vail, Colorado. Alphaherpesviruses include neurotropic viruses of humans (types 1 and 2 herpes simplex virus and varicella zoster virus), simian varicella virus of nonhuman primates, and significant pathogens affecting domestic meat and poultry industries (bovine herpesvirus, pseudorabies virus, and Marek's disease virus). The CALS mission is to facilitate studies in all aspects of alphaherpesvirus latency and reactivation by providing a venue where current research is presented for extensive discussion. The relaxed atmosphere provided in Vail is a quiet mountain resort located in the heart of the Colorado Rocky Mountains was the ideal setting for 39 established clinical and basic research scientists from around the world to describe their recent findings (Fig. 1). This year's CALS also featured a discussion of the technical advances in next generation DNA sequencing, including problems and pitfalls. A brief summary of each presentation follows.

\section{Reports}

Don Gilden, University of Colorado, presented studies that describe the incidence of varicella zoster virus (VZV) infection in temporal arteries (TA) of patients with clinically suspected giant cell arteritis (GCA), but with pathologically negative

R. J. Cohrs · D. Gilden $(\bowtie)$

Departments of Neurology, University of Colorado Denver School of

Medicine, Mail Stop B182, 12700 E. 19th Avenue, Aurora,

CO 80045, USA

e-mail: don.gilden@ucdenver.edu

R. J. Cohrs $\cdot$ D. Gilden

Department of Microbiology, University of Colorado Denver School of Medicine, Aurora, CO, USA
TAs. Immunohistochemical analyses of archived formalinfixed, paraffin-embedded TAs from such patients revealed VZV, but not herpes simplex virus-1 (HSV-1), antigen in five ( $21 \%$ ) of 24 patients, whereas similar analyses of 13 normal TAs from autopsied subjects over age 60 years revealed neither VZV nor HSV-1 antigen. All five patients whose TAs contained VZV antigen had presented with clinical and laboratory features of GCA and early visual disturbances. Multifocal VZV vasculopathy can present with the full spectrum of clinical features and laboratory abnormalities characteristically seen in GCA.

Georges M.G.M. Verjans, Erasmus Medical Center in Rotterdam, presented new data on the emergence of acyclovir (ACV) resistance in patients with recrudescent HSV-1 keratitis. Quantitation of ACV-resistant HSV-1 viruses in sequential corneal HSV-1 isolates from patients with a history of herpetic stromal keratitis demonstrated that long-term (>12 months) $\mathrm{ACV}$ prophylaxis results in emergence of ACV-resistant virus.

Marius Birlea, University of Colorado, described the clinical features, pathological changes, and immune repertoire in a remarkable case of virologically verified, chronic VZV ganglioneuritis without rash. The patient was a 39-year-old, morbidly obese man who experienced three episodes of maxillary distribution zoster during the last 2 years of life. Three months before death, he developed severe headache, followed by vertigo, word-finding difficulties, and recurrent maxillary distribution pain without rash. CT scan showed brain swelling; the CSF contained a mild pleocytosis, but no amplifiable VZV or HSV DNA. Headache worsened despite multiple medications, and the patient died in sleep. Forensic autopsy revealed mild cerebral edema with perivascular inflammation in the hippocampus. The hippocampus did not contain VZV or HSV antigen. Seven cerebral arteries around the circle of Willis as well as CSF removed at autopsy were negative for VZV and HSV DNA, and there were no anti-VZV antibodies in the 


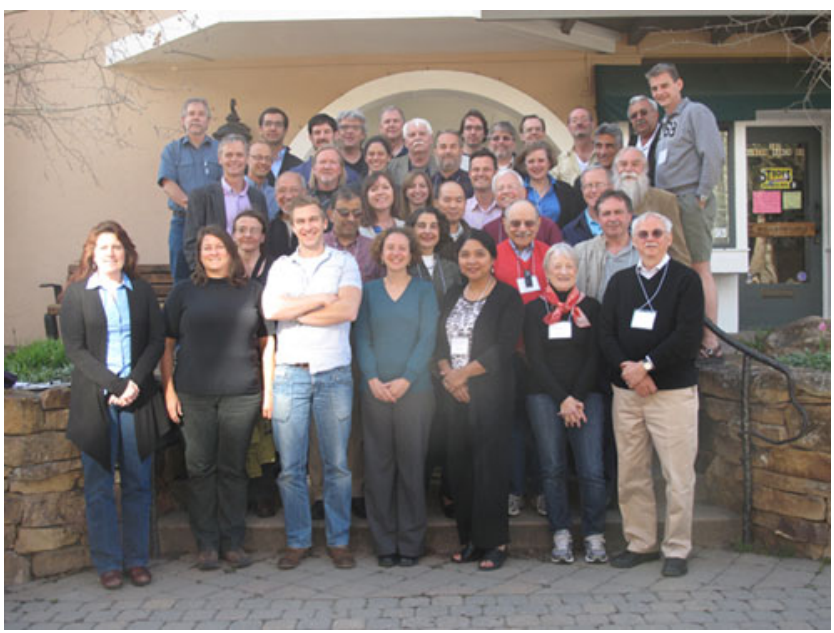

Fig. 1 Participants of the 2013 Colorado Alphaherpesvirus Latency Symposium. (left to right; row 1) Andrea Bertke, Leigh Zerboni, Werner Ouwendijk, Ilhem Messaoudi, Maria Nagel, Anne A Gershon, and Lynn Enquist; (row 2) Martine Aubert, Ravi Mahalingam, Vicki Traina-Dorge, Michael D. Gershon, and Stacey Efstathiou; (row 3) Luis Schang, Edouard Cantin, Stephanie James, Lora Shiflett, Jason Chen, Don Gilden, and Daniel Carr; (row 4) Kenneth Miller, David Bloom, Jessica Linderman, Charles Grose, Angus Wilson, David Tscharke, Judy Breuer, and Randy Cohrs; (row 5) Kenneth Jones, Marius Birlea, Nicholas Baird, Georges Verjans, Clinton Jones, Joao Proenca, Joel Rovnak, David Davido, Thomas Kristie, Todd Margolis, Homayon Ghiasi, and William Halford

CSF. PCR amplified VZV and HSV-1 DNA in both trigeminal ganglia. Histological examination revealed extensive inflammation in the ganglia and adjacent nerve roots. The inflammatory response was dominated by $\mathrm{T}$ cells $(\mathrm{CD} 4+>\mathrm{CD} 8+)$, and $\mathrm{CD} 68+$ macrophages/microglia were abundant; rare CD20+ B cells, CD15+ neutrophils, and granzyme B+ cells were also detected. Immunostaining revealed abundant VZV, but not HSV-1, antigen in the trigeminal nerve roots. The findings were compared with those of the only other reported case of chronic VZV ganglioneuritis without rash.

Michael Gershon, Columbia University, showed that VZV can infect the human gastrointestinal tract and become latent in enteric nervous system (ENS) neurons. Although VZV is not present in the ENS at birth, latent infection in adults is prevalent and may include almost all individuals who have experienced varicella or have been vaccinated against it. Moreover, transcripts encoding VZV ORF63 were found only in segments of gut that contain ganglia; for example, in two patients with Hirschsprung's disease, these transcripts were detected in proximal segments of colon containing enteric ganglia, but not in distal aganglionic segments of bowel. Dr. Gershon recently demonstrated that VZV reactivation caused severe gastric hemorrhage necessitating partial gastrectomy in a 16-year-old immunocompetent boy. DNA encoding vaccine type VZV genes (ORFs 40 and 67) was detected in tissue removed at surgery as well as in saliva, and VZV gE and ORF63 proteins were found by immunocytochemistry in epithelial cells at the lesion margins; importantly, no VZV gene products were found in repeat stomach biopsies after recovery. To further understand enteric VZV infection and its consequences, Dr. Gershon and colleagues have developed a guinea pig model. VZV was passed from infected human embryonic lung fibroblasts to guinea pig $\mathrm{T}$ lymphocytes in vitro. Infected lymphocytes expressed VZV immediateearly ORF62 protein and VZV late gE protein and produced virions as detected by electron microscopy. As in VZVinfected human cells, infection of guinea pig lymphocytes was cell associated and no filterable virus was detected. In guinea pigs injected with VZV-infected guinea pig T cells, VZV immediate-early and early transcripts were detected within 2 days in resident macrophages of liver, lung, and spleen. After 4 weeks, VZV disappeared from these organs but was detected in ENS and dorsal root ganglia, where only immediate-early genes were transcribed. Latent VZV within enteric neurons was confirmed by in situ hybridization, which revealed VZV immediate-early proteins transcripts detected by immunocytochemistry. Use of VZV expressing green fluorescent protein driven by the ORF66 promoter showed that VZV-infected lymphocytes administered intravenously into guinea pigs led to latency in virtually all enteric neurons and most dorsal root ganglia. Preliminary evidence suggests that treatment with corticotrophin-releasing hormone leads to VZV reactivation in the ENS and detection of VZV DNA in saliva. Future studies using the guinea pig model will investigate stimuli of VZV reactivation in vivo as well as the nature and pathophysiology of disorders arising from enteric zoster, a difficult diagnosis to make in the absence of rash.

Edouard Cantin, Beckman Institute City of Hope, presented findings on the use of pooled human immunoglobulins given intravenously (IVIG) to establish HSV latency in immunodeficient susceptible 129S6 (129) and resistant C57BL/6 (B6) recombination-activating gene (Rag)-deficient mice. IVIG promotes long-term survival and latency by a mechanism(s) involving suppression of intracellular virus replication and pathological CNS inflammatory responses. Rag mice inoculated with HSV-1 (strain $17+$ ) at or below $32 \times \mathrm{LD}_{50}$ (low-dose) were protected by a single dose of IVIG given $24 \mathrm{~h}$ post-infection (pi), whereas Rag mice inoculated at $100 \times \mathrm{LD}_{50}$ (high-dose) required at least three additional cycles of IVIG treatment every 12 days beginning at $24 \mathrm{~h}$ pi for survival and establishment of latency. Additionally, a 10-day course of acyclovir was given intraperitoneally beginning at 12 days pi to ensure elimination of replicating HSV. Random screening of latently infected mice over a 2-month period after the last IVIG treatment was negative for infectious HSV in trigeminal ganglia (TG) and brainstem. However, mice developed fatal HSV encephalitis (HSE) sporadically after high-dose, but not low-dose HSV in latently infected Rag mice and these high-dose mice had high levels of virus in both TG and brainstem. Consistent with this finding, in vivo reactivation 
of latent HSV induced by hyperthermic stress caused eye and periocular skin disease, culminating in high rates $(>70 \%)$ of fatal HSE only in high-dose mice. However, HSV could be reactivated in explant cultures of TGs from low-dose latently infected mice. Thus, $32 \times \mathrm{LD}_{50}$ represents the threshold inoculum that enables in vivo reactivation using mortality and/or eye disease as endpoints. Adoptive transfer of memory, but not naive $\mathrm{CD} 4+$ or $\mathrm{CD} 8+\mathrm{T}$ cells, into latently infected Rag mice $48 \mathrm{~h}$ before in vivo reactivation prevented reactivation. The high-dose is only threefold higher than the low-dose inoculum, and latent viral DNA loads in TGs determined by HSV-gG Taqman PCR did not differ in the two groups. Thus, the cause of reactivation in high-dose-inoculated mice remains unclear.

Luis Schang, University of Alberta, discussed atypical HSV-1 DNA chromatin in productive virus infection. The nucleosomes in HSV-1 chromatin form highly unstable structures that cannot fully block transcription activator access to viral DNA. All major HSV-1 transcription activators can induce chromatin remodeling. Initially, activation of viral transcription should mobilize histones. Then, as the virus genome replicates, increasing numbers of histones would be involved in the unstable viral chromatin, predicting a progressive increase in the percentage of histones not bound to chromatin in infected cells. In fact, the percentage of unbound linker and core histones increased at 4 and $7 \mathrm{~h}$ post-infection. HSV-1 with mutations in each of the major transcription activators (VP16, ICP0, or VP16) showed a reduced ability to induce the increase in unbound histones or to alter the rates of fast or slow histone chromatin exchange, indicating that transcription activators induce histone mobilization. However, the HSV-1 mutants did not express abundant early or late proteins, raising the possibility that defective histone mobilization arises indirectly from insufficient expression of these proteins. Inhibition of HSV-1 DNA replication by phosphonoacetic acid did not drastically affect histone mobilization, indicating that the mobilization is not a consequence of the fast rate of viral DNA synthesis in infected cells. Moreover, late proteins are not required for histone mobilization. HSV-1 with a mutation in ICP0 or VP16 mobilized histones, transcribed the genome, and replicated with near wild-type kinetics in U2OS cells. To test whether an unidentified early protein expressed in U2OS cells infected with these mutants might mediate the induction of histone mobilization by late HSV proteins, ICP4 was expressed in the absence of all other HSV-1 proteins from a transiently transfected expression vector; ICP4 alone was sufficient to induce mobilization of core histones. Overall, the dynamics of all linker and core histones are altered in cells productively infected with HSV-1. All three major HSV-1 transcription activators modulate mobilization of histones during infection, directly or indirectly. These results are consistent with the model in which a host antivirus response (epigenetic silencing virus gene transcription) is circumvented by virus- encoded transcription activators through mobilizing histones away from the viral genomes.

Thomas M. Kristie, NIH, presented an overview of the role of LSD1 and the JMJD2 family of histone demethylases in regulating early HSV infection via chromatin remodeling. After infection, the HSV genome is rapidly assembled into a canonical chromatin structure. Initially, the assembled chromatin is composed of nucleosomes with repressive histone modifications that suppress viral gene expression. To circumvent this repression and begin viral gene expression, viral and cellular transcriptional activators recruit a cellular coactivator (HCF-1) complex to the promoter domains of viral immediate-early genes. This complex contains multiple chromatin modification components, including the histone demethylases LSD1 and JMJD2s, which remove repressive histone H3lysine 9 methylation marks, and a histone methyltransferase (Set/MLL1), which installs the transcription-stimulating histone H3-lysine 4 methylation. Both classes of HCF-1associated histone demethylases (LSD1 and JMJD2 family proteins) were shown to be essential for reversing repression of viral immediate-early genes. Additionally, high-potency inhibitors of either LSD1 or JMJD2 histone demethylase activities blocked lytic viral infection and promoted continued repression of the viral genome. These inhibitors also blocked expression of immediate-early genes during HSV reactivation from latency in a mouse ganglia explant model. Because the coactivator HCF-1 has been proposed to be critical for both initiation of lytic infection and reactivation from latency, the data suggest that HCF-1-dependent chromatin modulation contributes to or is required for viral reactivation. Collaborative studies to investigate the impact of demethylase inhibitors in vivo, including studies in the mouse model of primary infection (Debra Quenelle, University of Alabama at Birmingham), the rabbit eye model of spontaneous reactivation (Jim Hill, Louisiana State University Eye Center, LSU School of Medicine), and the guinea pig model of recurrent genital HSV (Rhonda Cardin, Cincinnati Children's Hospital Medical Center) demonstrated reduction in primary infection along with suppression of viral reactivation and shedding. The in vivo results support the notion that chromatin modulation is critical in HSV infection and suggest the promise of novel antivirals that target chromatin machinery required for initiation of viral lytic infection and reactivation.

Clinton Jones, University of Nebraska. Bovine herpesvirus 1 (BHV-1) establishes a life-long latent infection in sensory neurons after acute infection. Increased corticosteroid levels due to stress increases reactivation. Within minutes of corticosteroid administration, the glucocorticoid receptor is activated and transcription is initiated from promoters containing a glucocorticoid receptor element. A single intravenous injection of the synthetic corticosteroid dexamethasone induced reactivation from latency in $100 \%$ of latently infected calves, and lytic cycle viral gene expression was detected within $6 \mathrm{~h}$ 
after dexamethasone treatment of calves latently infected with BHV-1. Cellular transcription factors were induced in trigeminal ganglionic neurons within $1.5 \mathrm{~h}$ after dexamethasone treatment, suggesting that they induced viral gene expression during early reactivation. Immunohistochemical analysis of viral protein expression during early reactivation consistently detected bICP0 and a late protein (VP16) in a subset of trigeminal ganglionic neurons within $1.5 \mathrm{~h}$ after dexamethasone treatment. Most neurons expressing bICP0 also expressed VP16. Neurons expressing the glucocorticoid receptor also expressed bICP 0 or VP16 at $1.5 \mathrm{~h}$ after dexamethasone treatment. Two other late proteins, glycoproteins $\mathrm{C}$ and $\mathrm{D}$, were not detected until $6 \mathrm{~h}$ after dexamethasone treatment and only in a few neurons. These studies provide evidence that VP16 and the promiscuous viral transactivator bICP0 are expressed early during reactivation, and that they stimulate extensive viral gene expression and production of infectious virus in a small subset of latently infected neurons.

Maria Nagel, University of Colorado, discussed intimal thickening, disruption of the arterial media, and molecular aspects of vascular remodeling in VZV-infected cerebral arteries that lead to stroke. Purinergic signaling mediated by ATP and its metabolites has been shown to be important for pathological vascular remodeling. To study the role of specific purinoreceptors in VZV infection and subsequent vascular remodeling, primary human brain vascular adventitial fibroblasts (BRAFs) were treated with antagonists of relevant purinoreceptors P2X1, P2X4, P2X7, P2Y2, P2Y6, P2Y11, and P2Y12. Treated and untreated cells were infected with $\mathrm{VZV}$, and viral DNA was quantified at 3 days post-infection by real time-PCR. Clopidogrel, a P2Y12 antagonist, downregulated VZV DNA by $70 \%$ compared to untreated controls, whereas other antagonists revealed no significant downregulation. Parallel DNA studies using clopidogrel on HSV-1- and HSV-2-infected BRAFs revealed $>99 \%$ reduction in viral DNA as compared to untreated cells in both groups. Corresponding viral titers reflected decreased viral DNA in clopidogrel-treated BRAFs compared to untreated BRAFs; similar growth curves were observed for HSV-1 and HSV-2. Immunocytochemical analysis using anti-VZV gE antibody showed that VZV-infected BRAFs treated with clopidogrel had decreased plaque number and size, as compared to untreated infected BRAFs in addition to aberrant cellular processes consistent with the known involvement of P2Y12 in lamellipodia formation. Overall, the findings suggest that (a) interference with P2Y12 purinoreceptor signaling in BRAFs attenuates VZV infection, as well as HSV-1 and HSV-2 infection and (b) the P2Y12 antagonist clopidogrel, which is already in clinical use as an antiplatelet agent, is a potentially effective antiviral agent.

Angus Wilson, New York University, addressed the mechanism regulating entry into latency by HSV proteins involved in initiating lytic (productive) infection. He noted the curious example of Kaposi's sarcoma-associated herpesvirus (KSHV), a gammaherpesvirus that shows strong preference for latency after infection. Unexpectedly and seemingly counterproductively, within the first few hours after infection, KSHV replication and transcription activator (RTA), a potent activator of many virus genes involved in productive infection also stimulates latency-associated genes. This initial wave of RTA expression stimulates transcription of latency-associated genes through a strong RTA-responsive element contained in the promoter located just upstream of the gene cluster. Accumulation of products from the latency-associated gene suppresses further RTA activity, allowing virus to establish and sustain latency. In sharp contrast, alphaherpesviruses use virus transactivating proteins (VP16-like initiator proteins) to initiate productive virus infection and inhibit latency in most cell types. The single exception to this paradigm is HSV infection of neurons, where VP16 is unable to inhibit latency. Preliminary studies showed that virus tegument-associated HSV VP16 delivered into the infected cells was either unable to translocate into the nucleus of infected neurons in culture or unable to transactivate virus gene transcription. Viruses were engineered to express an altered VP16 that failed to establish latency in neurons and instead replicated. Although the molecular basis for this profound switch in infection outcomes is unclear, the results indicate that VP16 can function as an activator when delivered into the neuronal cytoplasm and can also be suppressed by neuronal factors. Studies using chamber devices to isolate axons from neuronal cell bodies showed that wild-type viruses can establish latency in vitro in the absence of replication inhibitors, and that establishment of latency is most efficient when virus enters via axonal transport. Whether this holds true for viruses expressing the altered deregulated form of VP16 is currently under investigation.

David Davido, University of Kansas, discussed data on the HSV-1 immediate-early protein 22 (ICP22) which is required for efficient replication, virus-induced chaperone-enriched (VICE) domain formation and efficient expression of a subset of late virus proteins. ICP22 is also important for viral replication in vivo and establishment of latency. $U_{S} 1$, the gene that encodes ICP22, also produces a second protein that is an inframe N-terminally truncated form of ICP22 known as $\mathrm{U}_{\mathrm{S}} 1.5$. The respective functions of ICP22 and $\mathrm{U}_{\mathrm{S}} 1.5$ in viral infection have remained unclear. To address this question, viral mutants that express either ICP22 (M90A) or $\mathrm{U}_{\mathrm{S}} 1.5$ (3Xstop) were constructed. Both mutant viruses replicated to levels similar to that of wild-type HSV-1 in human embryonic lung cells and enhanced expression of late viral proteins, virus host shutoff, and $\mathrm{gC}$. However, unlike M90A, the 3Xstop mutant was unable to induce VICE domain formation in tissue culture or to replicate efficiently in the eyes and trigeminal ganglia of mice during acute infection or to become latent. Thus, while both ICP22 and $\mathrm{U}_{\mathrm{S}} 1.5$ can promote efficient replication and activate expression of late genes in certain cell types, only 
ICP22 induces VICE domain formation and enhances acute replication in vivo.

Lynn Enquist, Princeton University, presented data on his studies with pseudorabies virus (PRV). A clinical hallmark of alphaherpesvirus infections, including PRV, is peripheral pain or itching. While previous in vitro studies showed that cultured neurons infected with PRV exhibited aberrant electrical activity due to virus-induced membrane fusion, it is unclear whether this mechanism occurs in vivo and correlates with disease symptoms. Dr. Enquist's laboratory examined neuronal activity during a virulent PRV infection of anesthetized mice by imaging infected peripheral nervous system ganglia in vivo using virulent and mutant PRV recombinants expressing the genetically encoded fluorescent calcium indicator protein GCaMP3. This calcium sensor fluoresces green in the presence of calcium transients and thus correlates with neuronal activity. Calcium imaging enabled monitoring activity in vivo of multiple infected neurons simultaneously with single-cell resolution using two-photon microscopy. Infection with virulent PRV caused neurons to fire synchronously and cyclically, independent of input from presynaptic brainstem neurons. This activity was not observed after infection with an attenuated PRV recombinant used for circuit tracing or with PRV mutants lacking a viral protein required for membrane fusion or for sorting virions in axons. These data suggest that virulent PRV induces electrical coupling in non-myelinated axons in vivo, leading to synchronized neural activity in ganglia and contributing to the characteristic peripheral neuropathy.

David Tscharke, Australian National University, presented work from collaboration with Frank Carbone and Joel Ma, University of Melbourne, to determine transcription profiles in individual neurons latently infected with HSV-1. ROSAYFP mice were infected with HSV-1 expressing Cre recombinase using the flank zosteriform model. Infected and uninfected neurons in the same dorsal root ganglion were identified by fluorescence and captured by laser-dissection microscopy. Analysis of RNA by quantitative RT-PCR demonstrated that cells harboring latent virus were predominantly sensory neurons positive for $\operatorname{TrkA}^{+}$, so further analyses were restricted to this subset of neurons. HSV-1 latency-associated transcript RNA was detected in $\sim 90 \%$ of $\mathrm{YFP}^{+}$neurons, but more surprisingly, lytic viral genes (including ICPs 0, 47, 27, 6,8 , VP16 and $\mathrm{gB}, \mathrm{gD}$, and $\mathrm{gC}$ ) were also frequently detected in the same cells. YFP ${ }^{+}$neurons were roughly evenly grouped as neurons expressing: (a) no lytic virus gene, (b) at least one virus gene of each kinetic class, and (c) virus genes from one or two but not all three kinetic classes. Principle components analyses to examine expression of selected cellular genes showed that profiles differed between $\mathrm{YFP}^{-}$and $\mathrm{YFP}^{+}$neurons. Furthermore, the pattern of host gene expression differed between $\mathrm{YFP}^{+}$neurons that did and did not express HSV-1 lytic genes. Thus, HSV-1 latency is dynamic, with both virus and host cell genomes transcriptionally active. Even during apparently quiescent infection, host cells appear to respond constantly to the presence of HSV-1.

David Bloom, University of Florida, presented characterization of transcription upstream of the latency-associated transcript (LAT) promoter during lytic and latent HSV-1 infection in murine sensory ganglia. The analysis was aimed at identifying precursors of miRH1 and miRH6, two HSV miRNAs that have been identified upstream of LAT. This work identified a novel latent transcript, termed $A T A L$, which is a $1.8-\mathrm{kb}$ polyadenylated transcript whose promoter overlaps with the CTCF-insulator element (B1) and is transcribed on the same HSV-1 strand as LAT. ATAL is expressed during both lytic and latent infection, but more so during latency. It can be detected by Northern blot analysis and by in situ hybridization in latently infected ganglia from mice, rats, and humans. Strand-specific RNA hybridization revealed that during acute infection, ATAL was expressed mostly in neurons that do not express LAT, whereas during latency, ATAL was found mainly in the nucleus in both LAT+ and LAT- neurons. Importantly, recombinant HSV-1 of both strains $17+$ and KOS with deletions of the 202-bp core LAT promoter (17 $\Delta \mathrm{Pst}$ and KOS $\Delta \mathrm{Pst}$ ) eliminated virtually all LAT and ATAL expression. This finding highlights the need to use specific mutations that inactivate LAT and ATAL to determine whether phenotypes previously attributed to LAT are actually a function of ATAL.

Joao Proenca, University of Cambridge, pointed out that the study of sensory neurons latently infected with HSV-1 has been hindered by the lack of reliable markers to identify and purify latently infected cells. His laboratory previously described a system capable of marking latently infected cells that relies on the use of HSV-1 Cre-expressing recombinants to infect Rosa26R reporter mice. Dr. Proenca reported that using recombinant virus bearing Cre recombinase under the control of either latency-specific or strong heterologous promoters resulted in efficient LacZ activation and marked the latent cell reservoir. Use of Ai6 Cre fluorescent reporter animals to mark, visualize, and isolate live latently infected cells revealed that latent viral DNA loads range from 1 to $>1,000$ copies per cell that high viral DNA copy number is associated with increased reactivation, and that latent virus significantly impacts the host transcriptome.

Dan Carr, University of Oklahoma, presented preliminary data assessing an HSV-1 vaccine to reduce the inflammatory response initiated by challenge with the highly neurovirulent McKrae strain of HSV-1. In wild-type C57BL/6J mice that survive an $\mathrm{LD}_{50}$ of HSV-1 (10,000 plaque-forming units [pfu]/ cornea), virus elicits a robust inflammatory response in the cornea, with epithelial cell loss, infiltration of leukocytes, and neovascularization (defined as hem- and lymph-angiogenesis). In wild-type mice immunized with an ICP0 mutant (obtained from Dr. William Halford, SIU) and subsequently challenged with HSV-1 strain McKrae, $100 \%$ mice survived. This efficacy 
correlated with a reduction in infectious virus in the cornea and nervous system during acute infection and with absence of corneal neovascularization by day 30 postinfection (pi). Neovascularization of the cornea in nonimmunized wild-type mice that survived virus challenge was robust, with blood and lymphatic vessels traversing throughout the central cornea. In a comparative study using mice deficient in the type I interferon receptor (CD118 KO), non-vaccinated CD118 KO mice challenged with 10,000 pfu/ cornea HSV-1 died 5-6 days pi, whereas all vaccinated CD118 KO mice survived virus challenge and showed neovascularization in the cornea, although less pronounced than in non-vaccinated wild-type mice. The results suggest that vaccine is not dependent on a functional type I interferon pathway to provide efficacy as measured by survival and neovascularization.

Charles Grose, University of Iowa, investigated VZV infection of highly pure $(>95 \%)$ terminally differentiated neurons derived from pluripotent stem cells. Two weeks after infection, neurons appeared healthy and the cell culture medium lacked infectious virus. Cells contained VZV DNA as well as transcripts and proteins corresponding to VZV immediate-early, early, and late kinetic phases of replication. To determine whether a defect in the VZV late kinetic phase might explain the lack of infectious virus, two aspects of the late kinetic phase were examined in VZV-infected neurons: (a) transcription and expression of the true late VZV gC ORF14 and (b) capsid and enveloped virus structures. The results indicated extremely limited $\mathrm{gC}$ expression in a minority of VZV-infected neurons, whereas the early-late gE glycoprotein (ORF68) was abundantly expressed. Similarly, transcript analyses showed decreased $\mathrm{gC}$ transcription as compared with gE. Examination of viral structures by highresolution transmission electron microscopy revealed fewer virus particles than typically observed in VZV-infected cultured fibroblasts or melanoma cells. Furthermore, virus particles were aberrant in that most capsids in the nuclei lacked a dense (DNA-containing) core and most enveloped structures in the cytoplasm were light particles (virus envelopes without capsids). These results suggest a broad deficiency in late phase replication and viral assembly during VZV infection of cultured neuronal cells.

Judy Breuer, University College London, presented insights into the pathogenesis of VZV in its natural host gained through whole-genome sequencing of live-attenuated vOka vaccine and of virus isolated from rash arising after vaccination. Immunization with vOka vaccine prevents varicella (chickenpox) in children and susceptible adults, but causes rash in small numbers of recipients. Like wild-type virus, vaccine virus becomes latent and reactivates to cause herpes zoster and other neurological complications. Hybridizationbased methodologies were used to enrich vOka DNA obtained from skin lesions arising after immunization. Deep-sequencing of the DNA revealed a mixture of genotypes, and specific alleles segregating with vaccine strain VZV were recovered from vaccine rashes. The very low genetic diversity in any single skin lesion indicated the existence of a genetic bottleneck between vOka inoculation and lesion formation. In the most instances, a lesion originated from a single viral haplotype. New mutations, defined as polymorphisms not present in the original vaccine, occurred predominantly at low frequency, and none were present consistently, indicating that new mutations are not critical for vOka rash formation. In addition, no recombination between vaccine and wild-type VZV was observed. At known vaccine loci, the vaccine allele was present in the rashes at lower frequencies compared to the original vaccine, and there was evidence for selection of the wild-type allele in coding areas of the genome $(p<0.001)$. Importantly, selection rates for the ancestral (wild-type) allele in vaccine rashes were highly significant at nine individual loci that encode amino acid changes in ORFs $0,13,31,55$, and 62 , suggesting that these reversions are directly involved in vOka rash formation. Selection for the vaccine allele occurred at only one synonymous locus. The genotypes of virus isolated from rash that arise after reactivation cannot be distinguished from those of virus isolated from rash immediately after vaccination, precluding identification of neurotropic vOka variants. Based on the dates of vaccination and recovery of virus from varicella-like rashes (occurring $<42$ days post-inoculation), the vOka mutation rate was calculated to be $6 \times 10^{4}$ substitutions per site per annum. Applying this mutation rate to currently circulating wild-type virus, the time to the most recent common ancestor of modern day VZV clades was estimated to be $\sim 10,000$ years. While the number of mutations in varicellalike rashes was proportional to the time since inoculation such that mutation rates were closely related, the mutation rate for cases of vOka zoster decreased with time after vaccine inoculation. The simplest explanation for this result is the absence of VZV replication during latency. Thus, based on the mutation rate derived during active replication (i.e., from cases of varicella-like rashes), VZV latency is estimated to be established between 1 and 4 weeks after infection.

Martine Aubert, Fred Hutchinson Cancer Research Center, reported the use of rare-cutting endonucleases (homing endonucleases, HE) for targeted mutagenesis of essential HSV genes as a therapeutic approach to cure chronic/latent viral infection. In a model of latent HSV-1 infection, Dr. Aubert demonstrated in situ targeted mutagenesis of latent HSV genomes in HSV-latently infected cells exposed to both HSVspecific HE (HSV-HE) and Trex2, a 3' to 5' DNA exonuclease. Exposure of latently infected cells to HSV-HE and Trex2 resulted in a significant decrease in infectious virus production after reactivation. While amplicon sequencing from treated cells revealed small deletions in the HSV-1 DNA sequence 
targeted by HSV-HE, the mutation frequency remained modest. One possible explanation was the low accessibility of the latent viral genome due to its compact state characterized by association to histones with posttranslational modifications indicative of repressed (silenced) DNA. To test whether HSV-1 DNA configuration might impact HE-mediated mutagenesis, HSV-1-infected cells were exposed to HSV-HE in the presence of either trichostatin A, a histone deacetylase inhibitor that induces derepression of the viral episome, or interferon- $\alpha$ to increase viral genome repression. The mutation frequency in HE-treated cells exposed to trichostatin A was increased twofold, while HE-mediated mutagenesis in HE-treated cells exposed to interferon was inhibited compared to HE-treated cells in the absence of either drug. These studies provide the groundwork for further studies aimed at developing a cure for HSV infection by HE-mediated targeted mutagenesis of virus DNA in latently infected neurons.

IIhem Messaoudi, University of California Riverside, presented studies of the $\mathrm{T}$ cell response in rhesus macaques during acute and latent infection with simian varicella virus (SVV), a homolog of VZV. While clinical observations suggest that successful control of VZV during primary infection and reactivation in unvaccinated individuals is mediated by cellular rather than humoral immunity, a comprehensive analysis of the T cell response to the entire VZV genome remains to be conducted. Because intrabronchial inoculation of young rhesus macaques with SVV which recapitulates the hallmarks of acute and latent VZV infection in humans, the specificity of $\mathrm{T}$ cell responses during acute and latent SVV infection was examined. Animals generated a robust and broad T cell response directed against both structural and nonstructural SVV proteins during acute infection in bronchoalveolar lavage (BAL) and peripheral blood cells. During latency, T cell responses were detected only in BAL and were lower and more restricted than those found during acute infection. Interestingly, a small set of ORFs was immunogenic during both acute and latent infection in BAL. Given the close genomic relatedness of SVV and VZV, these immunogenic ORFs warrant further attention as a potential component of second-generation VZV vaccines that specifically boost $\mathrm{T}$ cell immunity.

William P. Halford, Southern Illinois University, investigated the feasibility of live-attenuated HSV-2 vaccines. One billion people are infected with HSV-2, and $>30$ million live with frequent and recurrent genital herpes. More than $\$ 100$ million have been invested in subunit vaccines based on HSV-2 glycoprotein $\mathrm{D}(\mathrm{gD}-2)$, an approach that has failed six clinical trials spanning 22 years and has led to doubts about the feasibility of a HSV-2 vaccine. While available evidence raises questions about viability of glycoprotein subunitbased vaccines, other vaccine modalities exist but have not been tested in clinical trials. Analysis of mice vaccinated with a construct mutated in the HSV-2 ICP0 nuclear location signal
(HSV-2 0deltaNLS), predicted by earlier HSV-1-based studies to be acutely interferon-sensitive and avirulent in vivo, acquired nearly sterilizing immunity against lethal HSV-2 challenge, whereas $<10 \%$ of gD-2-immunized animals survived the same rigorous HSV-2 challenge. Guinea pigs immunized with live HSV-2 0deltaNLS vaccine showed superior protection against subsequent HSV-2 challenge. Live HSV-2 OdeltaNLS vaccine elicited a polyclonal IgG response against total HSV-2 antigen that was 35-fold greater than that elicited by a gD-2 subunit vaccine, and the resulting antibody response was directed against at least $18 \mathrm{HSV}-2$ proteins. Thus, the calculated risk of immunizing humans with a live avirulent HSV-2 mutant virus may be preferable to the current situation in which a billion HSV-2 carriers continue to spread wild-type HSV-2 infections to $\sim 20$ million naïve individuals per year.

Homayon Ghiasi, Cedars-Sinai Medical Center, investigated the contribution of immune cells to the establishment of HSV-1 latency. HSV-1 infection is a classic example of chronic/latent persistent viral infection in both human and experimental animal models. $\mathrm{CD} 8^{+} \mathrm{T}$ cells have been shown to infiltrate trigeminal ganglia (TG) at the time latency is established, where they inhibit HSV-1 reactivation. To determine whether $\mathrm{CD} 8^{+} \mathrm{T}$ cells or $\mathrm{CD} 8 \alpha^{+}$dendritic cells (DCs) contribute to greater latency and reactivation frequencies in ocularly infected mice, latency in TG of wild-type, $\mathrm{CD} 8 \alpha^{-/-}$ and $\mathrm{BXH} 2$ mice was examined. Both $\mathrm{CD} 8 \alpha^{-/-}$and $\mathrm{BXH} 2$ mice lack $\mathrm{CD} 8 \alpha \mathrm{DCs}$, but $\mathrm{CD} 8 \alpha^{-/}$mice also lack $\mathrm{CD} 8^{+} \mathrm{T}$ cells, while $\mathrm{BXH} 2$ mice have a full complement of $\mathrm{CD} 8^{+} \mathrm{T}$ cells. Additionally, $\beta 2 \mathrm{~m}^{-/-}$and $\mathrm{CD} 8 \beta^{-/-}$mice, which have $\mathrm{CD} 8 \alpha$ DCs, were examined. Experiments were also conducted to determine whether DCs from these mice behave differently after infection with $\operatorname{LAT}(+)$ or LAT $(-)$ viruses and whether the phenotype of $\mathrm{CD} 8 \alpha^{--}$and $\mathrm{BXH} 2$ mice could be restored to that of wild-type mice by adoptive transfers of wild-type $\mathrm{CD} 8^{+}$ T cells or wild-type bone marrow-derived CD $8 \alpha$ DCs. Overall, HSV-1 latency was significantly decreased in vivo in the absence of $\mathrm{CD} 8 \alpha^{+} \mathrm{DCs}$ but not $\mathrm{CD} 8^{+} \mathrm{T}$ cells. The findings indicate a key role for $\mathrm{CD} 8 \alpha^{+} \mathrm{DCs}$ and not $\mathrm{CD} 8^{+} \mathrm{T}$ cells in establishment and maintenance of HSV-1 latency in TG of infected mice.

Werner J.D. Ouwendijk, Erasmus Medical Center in Rotterdam, discussed studies using SVV to identify host cell types involved in virus dissemination during primary infection. African green monkeys were inoculated intratracheally with wild-type SVV and recombinant SVV expressing enhanced green fluorescent protein (SVV-EGFP). By visualizing SVV-EGFP-infected cells in living animals and in tissue samples, virus-infected cell types in blood, lungs, skin, and ganglia during primary infection were identified. During viremia, SVV predominantly infected peripheral blood memory T cells. Detection of SVV-infected memory T cells in lungs, in 
early varicella skin lesions, and to a lesser extent in ganglia suggests a role for these cells in transporting virus to these organs.

Kenneth Jones, University of Colorado, presented RNA sequencing data derived from VZV-infected human neurons in culture. VZV, an exclusively human neurotropic alphaherpesvirus, has a unique non-lytic relationship in neurons in contrast to productive infection of cells from other organs. Highly pure $(>95 \%)$ terminally differentiated human neurons derived from VZV-infected pluripotent stem cells appear healthy 2 weeks later and do not release infectious virus into the culture medium, but contain virus DNA, transcripts, and proteins. To further characterize nonproductive infection in human neurons, RNA deep-sequencing techniques were used to examine the entire VZV transcriptome. Total RNA was extracted from VZV-infected human fetal lung fibroblast cells at the height of a cytopathic effect $(n=2)$ and from VZV-infected terminally differentiated human neurons 14 days post-infection $(n=2)$. Illumina HiSeq 2000-based sequencing results were obtained and transcript levels were quantified in fragments per kilobase of exon per million mapped reads via CUFFLINKS, followed by statistical analysis (ANOVA) of differential gene transcription. Within infected neuronal cells, various viral genes were downregulated, most notably several genes that encode capsid, envelope, and tegument proteins. These results indicate that VZVinfected neurons are defective in late-phase virus gene transcription, and that insufficient structural proteins are produced to assemble intact virons.

Acknowledgments The Colorado Alphaherpesvirus Latency Society thanks Abcam, Perkin Elmer, Beckman Coulter, Miltenyi Biotec, and the VZV Foundation for their generous support. We especially thank the owners of the Christiania Lodge at Vail. 